\title{
A search for transiting planets in the Galactic Plane
}

\author{
Veronica R. Miller and Michael D. Albrow \\ Department of Physics and Astronomy, University of Canterbury, \\ PO Box 4800, Christchurch, New Zealand \\ email: veronica.miller@pg.canterbury.ac.nz
}

\begin{abstract}
We are carrying out a deep survey for transiting extrasolar planets in a 1 square degree field in the Galactic Plane. The images to date were taken using the Wide Field Imager on the ESO $2.2 \mathrm{~m}$ telescope at La Silla. We present details of the analysis and initial results from the survey.
\end{abstract}

\section{Dataset and Observations}

All images were taken using the ESO 2.2m telescope on La Silla, Chile using the Wide Field Imager instrument (WFI). Each WFI image is a mosaic of $82 \mathrm{k} \mathrm{x} 4 \mathrm{k}$ CCD images, covering a 1 square degree field.

Initial observations consisted of 63 images of a single 1 square degree field in the Norma spiral arm that were taken in 2002 by the PLANET collaboration (Albrow et al. (1998)). The observations had irregular temporal spacing due to their acquisition during a campaign of observations of microlensing events. In 2005, further observations of the same field with the same instrumentation were acquired in ESO service observing mode (P.I. C. Afonso). This second set of data is more regularly spaced in time and consists of 204 images, bringing the total number of observations to 267.

\section{Reduction}

A variant of the PySIS3 software (Albrow (2008)) was used for the reduction. This code implements the image subtraction method of Alard \& Lupton (1998) but uses a numerical pixel grid (Bramich (2008)) rather than a sum of Gaussian functions to define the kernel. Initially a deep reference template was created from 3 of the images with the best seeing. This template was mapped to and subtracted from each individual image. To perform these procedures, the best results were obtained by first splitting each CCD frame into 8 $1 \mathrm{k} \times 1 \mathrm{k}$ squares and reducing each of these separately and allowing each kernel to have a bi-quadratic spatial variation. The kernel size was also scaled to match the seeing. The DAOphot II code Stetson (1987) was used to detect and measure the fluxes of more than 500,000 stars on the reference image. PSF photometry of the difference images was performed for all detected stars using PySIS3.

\section{Lightcurves}

Initially simple cleaning was performed using the RMS of the lightcurves to remove extraneous values, following this use was then made of the trend filtering algorithm proposed by Kovács and Bakos (2007). Fig. 1 shows the change to the RMS of the lightcurves made by the cleaning and detrending processes and the comparison to Poisson noise. 


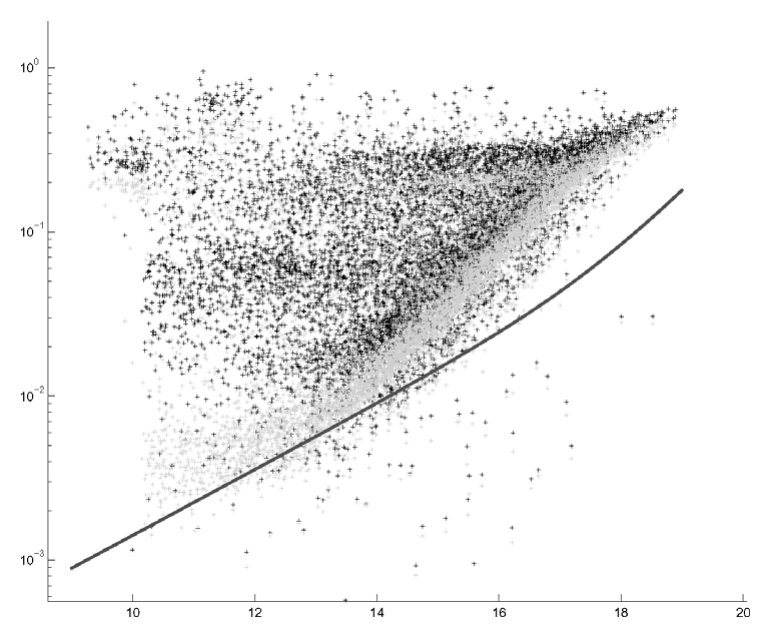

Figure 1. Change to RMS of lightcurves by means of cleaning and detrending as compared to the Poisson noise

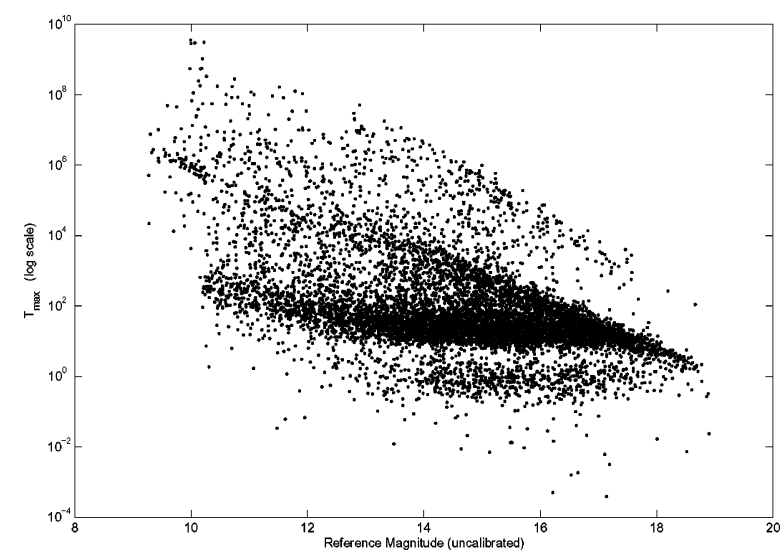

Figure 2. Results from using the transit algorithm on the cleaned lightcurves

\section{Search Algorithm}

For our algorithm, a modified grid search, we utilise the maximised matched filter as our test statistic (Tingley 2003a, Tingley 2003b). Rather than using a set of nested loops to cover the parameter space at some particular phase resolution, a matrix formalism has been used to test every possible set of in-transit points for a given trial period. The use of a sparse matrix in MATLAB allows a considerable improvement in speed over the traditional grid search. For each set of trial periods we compute the test statistic, Equation 4.1, for all possible sets of in-transit points. Fig. 2 shows the results from running the transit search algorithm on a selection of lightcurves. Fig. 3 contains the phased lightcurves resulting from the transit search algorithm of three stars which show some indication of structure in the lightcurve.

$$
T=d\left(\sum_{n=\text { intransit }} \frac{x_{n}}{\sigma_{n}^{2}}\right)-\frac{1}{2} d^{2}\left(\sum_{n=\text { intransit }} \frac{1}{\sigma_{n}^{2}}\right)
$$



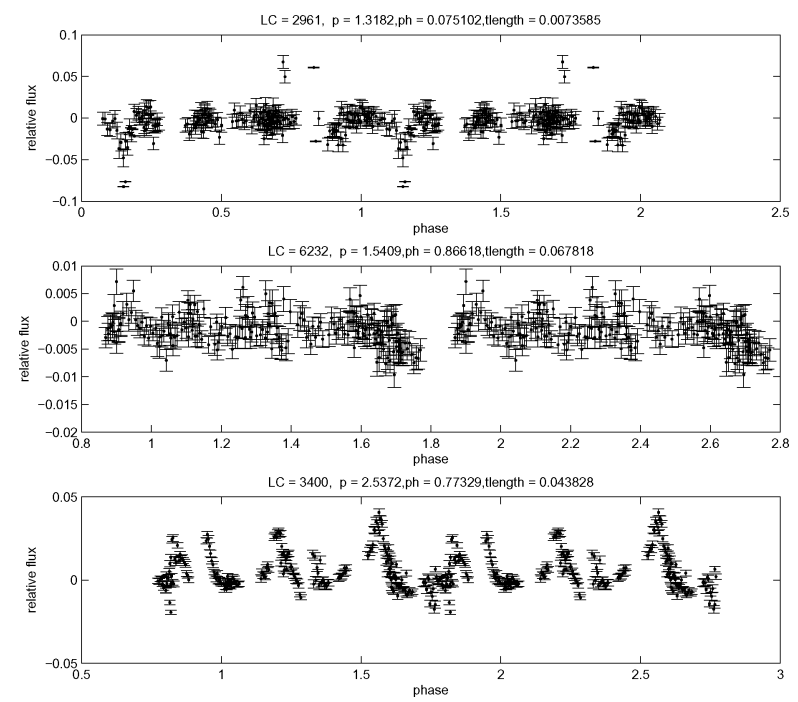

Figure 3. Lightcurves of three stars with results from the search algorithm above the search threshold.

\section{Further work}

We are currently in the process of searching the database for variable stars and transit candidates. The variable stars will be characterised and catalogued. We expect up to 10 transiting planets to be discoverable in the sample.

\section{References}

Alard, C. \& Lupton, R. H. 1998, ApJ, 503, 325

Albrow, M. D., Beaulieu, J-P., Birch, P., Caldwell, J. A. R., Kane, S., Martin, R., Menzies, J., Naber, R. M., Pel, J-W., Pollard, K., Sackett, P. D., Sahu, K. C., Vreeswijk, P., Williams, A., \& Zwaan, M. A. 1998, ApJ, 509, 687

Albrow, M. D. 2008, In Preparation

Bramich, D. 2008, In Preparation

Kovács, G. \& Bakos, G. Á. 2007, in: C. Afonso, D. Weldrake \& T. Henning (eds.), Transiting Extrasolar Planets Workshop, ASP-CS, 366, 133

Stetson, P. B. 1987, PASP, 99, 191

Tingley, B. 2003a, $A \& A$, 403, 329

Tingley, B. 2003b, $A \& A$, (Letters) 408, L5 